\title{
KRINKO-Empfehlungen zum Infektionsmanagement
}

\author{
Heinz-Michael Just
}

Wie in den beiden vorangegangenen Heften, so steht auch in diesem die Kommission für Krankenhaushygiene und Infektionsprävention (KRINKO) im Mittelpunkt. Das ist auch gut so, denn schließlich ist sie in $\S 23$ Abs. 2 des Infektionsschutzgesetzes (IfSG) als die Kommission genannt, die - angesiedelt beim und betreut vom Robert-Koch-Institut (RKI) - „Empfehlungen zur Prävention nosokomialer Infektionen sowie zu betrieblich-organisatorischen und baulich-funktionellen Maßnahmen der Hygiene in Krankenhäusern und anderen medizinischen Einrichtungen“ erstellen soll. Und das möglichst evidenzbasiert - was nichts anderes bedeutet als wissenschaftlich fundiert, abgesichert und praxisnah - also maximal verlässlich? Alle Mitglieder der KRINKO arbeiten ehrenamtlich und sind entsprechend motiviert, Empfehlungen zu veröffentlichen, die auch in Zeiten knapper Kassen eingehalten werden oder gerade in solchen - weil damit, so haben Studien gezeigt, Geld eingespart werden kann. Aber nur dann, wenn die Verantwortlichen in medizinischen Einrichtungen die erforderlichen Voraussetzungen geschaffen haben und die verantwortlichen Mitarbeiter qualifiziert sind.

Nicht immer jedoch lassen sich die wissenschaftlichen Kriterien für eine Evidenz bei allen Empfehlungen erfüllen, sei es, dass es kaum möglich ist, eine entsprechende randomisierte Studie durchzuführen, sei es, dass das Thema so grundlegend allgemein ist, dass eine Studie nicht zu bewältigende Dimensionen annehmen würde.

Vor einem solchen Dilemma stand die KRINKO bei der Neubearbeitung ihrer Basisempfehlung über die erforderlichen Grundlagen und Voraussetzungen, damit in medizinischen Einrichtungen für Patienten - aber auch für die Mitarbeiter! - die notwendige Sicherheit gegen Infektionsübertragungen verwirklicht werden kann. In 2 Beiträgen in diesem Heft werden die Details erläutert, warum die Entwicklung der letzten Jahrzehnte nicht nur den medizinischen Einrichtungen einen derart gestiegenen Bedarf an infektiologischem Know-how beschert hat - wo man doch vor 40 Jahren die Parole ausgab, durch die von der Industrie bereitgestellten
Antibiotika hätten Infektionen für immer ihren Schrecken verloren. Die Erreger dieser Infektionen waren, wie nicht anders zu erwarten war, schlauer. Sie zeigen uns derzeit mit den weltweit steigenden Antibiotikaresistenzen der Bakterien und den faszinierenden GenVariationen der Viren, wer wirklich klein ist - nicht die Mikroben! Die beeindruckend unwissenschaftliche Hysterie während der Vogelgrippe-Periode wie auch die teilweise merkwürdigen Verhaltensmuster der „Experten“ im Verlauf der neuen sogenannten Schweinegrippe machen deutlich, wie weit wir vom souveränen Umgang mit den unsichtbaren „Kleinen“ entfernt sind. Was wäre wohl passiert, wenn der neue Influenzatyp ein echtes aggressives Pandemiepotential gehabt hätte? Alle Pandemiepläne hätten letztlich versagt weil die darin theoretisch angemahnten Verhaltensweisen nicht umgesetzt worden wären, wegen z.T. engstirnigem Beharren auf Formalien und propagierter eigener Nicht-Zuständigkeit maskiert hinter der stereotypen Frage, wer das denn bitte alles bezahle.

Wie sieht es aus im kleineren Bereich der nosokomialen Infektionen? Auch dort werden gerne Forderungen umso überzeugter vertreten, je weiter sie von einem selbst angesiedelt sind und wenn sie einen selbst nicht fordern. Wir bauen lieber Schleusen und teure Klimaanlagen statt uns die Hände zu desinfizieren, wenn es nötig wäre! Trotz erdrückender Evidenzen! Wir berauben Patienten ihrer Freiheit, indem wir sie in Einzelzimmern „isolieren“, und wir gefährden sie damit zumindest potenziell, wie Untersuchungen eindrucksvoll gezeigt haben, nur weil wir bei ihnen Bakterien nachgewiesen haben, für die nicht diese Patienten verantwortlich sind, sondern letztendlich wir Ärzte. Und wenn unsere Verhaltensmuster kritisch hinterfragt werden (z. B. die missbräuchliche Verabreichung breit wirksamer Antibiotika ohne medizinische Indikation), dann ist für uns die stärkste Evidenz unsere angeblich so umfassende Erfahrung, genannt „Expertenmeinung“, ohne zu bedenken, dass eben diese der Motor unseres bisherigen Handelns und damit die Wurzel allen Übels war, bzw. ist. 
Deshalb haben sich die Anforderungen an Mitarbeiter, die sich der Infektionsprävention verschrieben haben, über die Jahre massiv verändert. Sie haben zugenommen und sind ganz nahe an den Patienten herangerückt. Krankenhaushygiene ist von einer eher im theoretischen Bereich der Medizin angesiedelten Disziplin zu einem Kernfach humanmedizinischer Versorgung geworden, über die Grenzen stationärer Einrichtungen hinaus. Moderne Krankenhaushygiene umfasst die ganze Bandbreite infektiologischer, klinischer, technischer, baulicher und umweltmedizinischer Aspekte und stellt damit die fachübergreifendste Disziplin in der Medizin dar. Dem musste die neue Empfehlung der KRINKO gerecht werden, ohne auf umfangreiche, gut konzipierte Studien zu Detailaspekten zurückgreifen zu können. Ich glaube, es ist ihr gelungen.

Trotzdem ist es eine Empfehlung - nicht mehr und nicht weniger. Und das soll sie auch sein. Denn Empfehlungen dieser Art regen notgedrungen dazu an, sich mit der eigenen Situation zu befassen und das Infektionsrisiko im eigenen Umfeld zu ermitteln und daraufhin zielorientiert zu handeln. Vorschriften bergen demgegenüber meist die Gefahr einer gedankenlosen Textbefriedigung, ohne dass dies das eigene Handeln nachhaltig beeinflussen muss.

Die KRINKO als einziges gesetzlich bestimmtes, Empfehlungen gebendes Expertengremium in Sachen nosokomiale Infektionen geht in ihrer exponierten Stellung in Zeiten zunehmenden kritischen Hinterfragens - sei es im Rahmen einer Zertifizierung oder einer Akkreditierung - aber auch eine hohe Verpflich- tung an ihre eigene Arbeitsweise ein. Dies erfordert Zeit, Disziplin und die Bereitschaft zu eher objektivanalytischen denn subjektiv-überzeugten Formulierungen.

Ein veränderter Kenntnisstand erfordert die Anpassung von Empfehlungen. Nie wurde das in so kurzer Zeit so eindrucksvoll deutlich wie bei der sogenannten „Schweinegrippe“. Fast täglich änderten sich die Empfehlungen und wurden dem Verlauf angepasst. Das fordert nicht nur diejenigen, die sie erarbeiten müssen, sondern auch die Betroffenen vor Ort. Bei der Bandbreite von Empfehlungen zur Infektionsprävention in medizinischen Einrichtungen ist die KRINKO vor eine nicht gerade kleine Aufgabe gestellt, der sie nach Kräften nachzukommen bemüht ist. Dass bei der Überarbeitung der alten und der Erarbeitung neuer Empfehlungen die Prioritäten nicht von allen gleich gesehen werden, ist hierbei nichts Ungewöhnliches.

Alle Kommissionsmitglieder sind bestrebt, dieser Aufgabe in möglichst kurzer Zeit gerecht zu werden, dabei alle Aspekte und Studien zu berücksichtigen, und die Erkenntnisse in praxisnahe und anwender- wie auch patientenfreundliche Empfehlungen umzusetzen. Jede konstruktive Kritik ist hierbei hilfreich, denn sie zeigt, dass die Empfehlungen Grundlage des Handelns geworden sind, und motiviert damit die Kommissionsmitglieder, ihre Empfehlungen immer auf dem aktuellen Stand zu halten. Hier, das haben die Beiträge der Kollegin Frau Professor Kappstein gezeigt, ist trotz aller Anstrengungen nach oben hin durchaus noch Luft. 